\title{
Otitis Externa, CTCAE
}

National Cancer Institute

\section{Source}

National Cancer Institute. Otitis Externa, CT CAE. NCI Thesaurus. Code C143719.

A disorder characterized by an infectious process involving the outer ear and ear canal.

Contributory factors include excessive water exposure (swimmer's ear infection) and cuts

in the ear canal. Symptoms include fullness, itching, swelling and marked discomfort in the ear and ear drainage. 